\title{
Editorial
}

\section{Virotherapy for cancer: Current status, hurdles, and future directions}

Cancer Gene Therapy (2002) 9, 959-960 doi:10.1038/sj.cgt.7700554

$\mathrm{T}$ his special issue on cancer virotherapy will review the emerging field of tumor-targeted viruses. The clinical effectiveness of any cancer treatment depends on both its antitumoral potency and the therapeutic index between cancerous and normal cells. Most current nonsurgical therapies for solid tumors fail on one or both scores, and it is unlikely that changes in dose or frequency, or even the combination of standard cytotoxic chemo - or radiotherapies will satisfy both of these standards. Metastatic cancer is currently treated with agents from four major "platforms": (1) chemotherapy (e.g., cisplatin, taxanes, etc.), (2) cytokines (e.g., interleukin-2, interferon-alpha), (3) monoclonal antibodies (e.g., rituximab, Herceptin), and (4) targeted small molecules (e.g., Gleeves, Imessa). Novel therapeutic approaches are required that offer greater potency and selectivity and that act by mechanisms that are not subject to cross-resistance when combined with standard therapies. Replication-selective oncolytic viruses fit these criteria. Replication in tumor tissue allows for a massive amplification of the input "dose" (e.g., 1000 - to 10,000-fold increases ) at the tumor site. Cancer cells are turned into in situ factories producing thousands of copies of the therapeutic agent; these viruses will then kill the "producer" cell and be released to attack neighboring cancer cells. In contrast, replication in normal tissues is abrogated leading to efficient clearance and reduced toxicity. Mechanisms leading to cancer selectivity include the (1) use of inherently selective RNA viruses, some of which take advantage of tumor cell resistance to interferon (e.g., measles virus vaccine strain MV-SPUD, reovirus, Newcastle Disease virus, NDV, vesicular stomatitis virus ); (2) deletion of viral genes that are necessary for replication in normal cells but expendable in cancer cells (vaccinia virus, adenovirus, herpes simplex virus, HSV, poliovirus); and (3) tissue-specific transcriptional regulatory elements driving viral genes that are critical for replication (adenovirus, HSV).

This special issue is timely for the following reasons. Firstly, this field is growing at an incredible rate. The number of engineered virus species entering or nearing clinical trials has gone from 1 in 1996 (adenovirus, dl1520) to roughly 10 in 2002; in addition to adenoviruses (e.g., Onyx015, CG7870, and CG7060), development candidates include HSV (G207, NV1020), reovirus (Reolysin), measles virus (MV-SPUD), vaccinia virus (e.g., tk-/vaccinia growth factor-negative), NDV (e.g., PV701), poliovirus, vesicular stomatitis virus (VSV). Secondly, clinical trial data are now available for several of these agents and initial hypotheses are being generated. Hurdles to this therapeutic approach have been identified and potential solutions proposed. Finally, it is now clear that this field poses unique biosafety and regulatory issues that are being addressed.

Several of the articles in this special issue will review preclinical and/or clinical data from specific virus species. $R N A$ viruses will be covered by Russell ${ }^{1}$ whereas $H S V$ is covered by Varghese and Rabkin. ${ }^{2}$ Specific aspects of adenovirus clinical data will be reviewed by Reid et $\mathrm{al}^{3}$ and Nemunaitis and Edelman. ${ }^{4}$ Bartlett $^{5}$ and Mastrangelo and Lattime $^{6}$ will review preclinical and clinical data, respectively, on vaccinia virus (and other poxviruses). In addition, novel approaches to enhance the systemic delivery of these agents to tumors will be reviewed by Green and Seymour. ${ }^{7}$ Ochsenbein ${ }^{8}$ reviews the use of viral agents to induce antitumoral immunity. Hermiston ${ }^{9}$ will review the field of "armed" virotherapy in which oncolytic viruses are engineered to express therapeutic transgenes by combining the best features of viral and gene therapy for cancer, multimodality as treatments result. Wisher ${ }^{10}$ reviews the critical field of release testing for replication-selective viruses before their use in clinical trials. Finally, unique biosafety and regulatory issues to consider for these agents are addressed by Vile et $\mathrm{al}^{11}$ in the final article.

Replication-selective viruses will be developed and eventually approved as products to improve the lives of cancer patients. It is unlikely that any single therapeutic agent will cure the most resistant solid cancers anytime soon. The stunning success of Gleevec (Novartis, Basel) in chronic myelogenous leukemia (CML) and gastrointestinal stromal tumors (GIST) may have more to do with the unique genetic nature of these tumors rather than the nature of this specific molecule. Whereas these tumors appear to be driven by a select few mutations early in their evolution (e.g., bcrabl or c-kit, respectively), most common carcinomas (e.g., lung, colon, breast, prostate) have hundreds or even thousands of mutations at the time of diagnosis. It is therefore not surprising that in contrast to the Gleevec success in CML, epidermal growth factor receptor inhibitors appear to have much less activity in common solid cancers. True clinical success and cures will certainly require combinations of rationally designed agents with each other and with standard treatments (e.g., chemotherapy, radiotherapy). Virotherapy agents will become an important part 
of the oncologist's armamentarium with which to fight a winning battle against cancer.

\section{David Kirn, MD}

Guest Editor

\section{References}

1. Russell SJ. RNA viruses as virotherapy agents.

2. Varghese S, Rabkin S. Oncolytic herpes simplex virus vectors for cancer virotherapy.

3. Reid T, Warren R, Kirn D. Intravascular adenoviral agents in cancer patients: lessons from clinical trials.
4. Nemunaitis J, Edelman J. Selectively replicating viral vectors. 5. Bartlett D. Poxvirus for virotherapy: translational research.

6. Mastrangelo MJ, Lattime EC. Virotherapy clinical trials for regional disease: In situ immune modulation using recombinant poxvirus vectors.

7. Green NK, Seymour LW. Adenoviral vectors: systemic delivery and tumor targeting.

8. Ochsenbein AF. Principals of tumor immunosurveillance and implications for immunotherapy.

9. Hermiston T. Gene delivery from replication-selective viruses: arming guided missiles in the war against cancer. J Clin Invest. 2000;105:1169-1172.

10. Wisher MH. Biosafety and product release testing issues relevant to replication-competent oncolytic viruses.

11. Vile R, Ando D, Kirn D. Scientific, regulatory, and biosafety hurdles for virotherapy agents. 